\title{
Sobolev 圆盘代数上的乘法算子*
}

\author{
王宗尧 ${ }^{* *}$ 刘义强
}

(华东理工大学数学系, 上海 200237)

\begin{abstract}
摘要 研究一类由单位圆盘 $D$ 上的 Sobolev 空间 $W^{2,2}(D)$ 中的解析函数构成 的代数, 称之为 Sobolev 圆盘代数, 给出了其上的有界线性乘法算子 $M_{f}$ 的基本 性质，刻画了乘法算子 $M_{f}$ 的换位子代数，证明了 $\mathcal{A}^{\prime}\left(M_{f}\right)$ 是交换的当且仅当 $M_{f}^{*}$ 是指标为 1 的 Cowen-Douglas 算子.
\end{abstract}

\section{关键词 Sobolev 圆盘代数 乘法算子 换位子}

Dixmier 和 Foiaç ${ }^{[1]}$ 首先基于 Sobolev 空间理论构造了有界线性算子模型, Herrero, Taylor 和王宗尧 ${ }^{2]}$ 将此模型用于对有界线性算子在紧摄动下点谱变化的 研究上. 以后王宗尧 ${ }^{[3]}$ 专门研究了 Sobolev 空间上的乘法算子, 并和蒋春澜一起 用于研究 Hilbert 空间上的强不可约算子 ${ }^{[4,5]}$. 虽然与 Sobolev 空间上算子有关的 一些算子代数有较好的性质, 例如严格循环性等, 但因为 Sobolev 空间上特殊的 内积定义使进一步研究遇到困难. 另一方面对单位圆上的 $H^{2}$ 和 $H^{\infty}$ 空间或者对 全体在单位圆 $D$ 内解析、 $\bar{D}$ 上连续的全体函数组成的圆盘代数的研究, 形成了 函数论中内容丰富的经典理论. 基于上述考虑, 本文研究了圆盘 $D$ 上的 Sobolev 空间函数所形成的函数代数, 本文称之为 Sobolev 圆盘代数, 及其上的乘法算子 的性质.

本文首先讨论 Sobolev 圆盘代数 $R(D)$ 及其上的乘法算子的一些基本性质, 例如规范正交基、再生核、 $R(D)$ 中函数的特征、作为 Cowen-Douglas 算子共轭的 乘法算子的谱图像和本性正常性等. 可分的复的无穷维 Hilbert 空间上有界线性 算子 $T$ 或一个算子类的换位子代数常常是人们关注的对象, 不同的算子或算子 类的换位子代数有不同的性质, 研究这些性质反过来对刻画这个算子或算子类的 结构会起很重要的作用. 本文刻画了 Sobolev 圆盘代数上乘法算子 $M_{f}$ 的换位子

2005-03-28 收稿

* 国家自然科学基金资助项目 (批准号: 10071020)

** E-mail: zywang@ecust.edu.cn 
代数 $\mathcal{A}^{\prime}\left(M_{f}\right)$, 并证明 $\mathcal{A}^{\prime}\left(M_{f}\right)$ 是交换的当且仅当 $M_{f}^{*}$ 是指标为 1 的 Cowen-Douglas 算子. 另外, 我们还给出了 $M_{z^{2}}$ 的所有 Banach 约化子空间.

\section{1 基本性质}

设 $\Omega$ 为复平面上的解析 Cauchy 域, 用 $W^{2,2}(\Omega)$ 表示 Sobolev 空间: $W^{2,2}(\Omega)=\left\{f \in L^{2}(\Omega, d m): f\right.$ 的广义函数的一阶、二阶偏导数在 $L^{2}(\Omega, d m)$ 中 $\}$, 其中 $d m$ 表示平面 Lebesgue 测度. 对 $f, g \in W^{2,2}(\Omega)$, 定义

$$
\langle f, g\rangle=\sum_{|\alpha| \leqslant 2} \int D^{\alpha} f \overline{D^{\alpha} g} d m,
$$

则 $W^{2,2}(\Omega)$ 成为一个 Hilbert 空间, 并且在一个等价范数下是具有单位元的 Banach 代数. 由 Sobolev 嵌入定理可知, $f \in W^{2,2}(\Omega)$ 蕴含着 $f \in C(\bar{\Omega})$, 并且存在 $M>0$, 使得 $\|f\|_{C(\bar{\Omega})} \leqslant M\|f\|_{W^{2,2}(\Omega)}$, 因此一列函数 $\left\{f_{n}\right\}_{n=1}^{\infty}$ 在 $W^{2,2}(\Omega)$ 中收玫到 $f$ 蕴 含 $f_{n}$ 在 $\bar{\Omega}$ 上一致收玫到 $f$. 关于 Sobolev 空间的更多知识可参考文献 [6].

对 $f \in W^{2,2}(\Omega)$, 我们定义 $W^{2,2}(\Omega)$ 上的乘法算子 $M_{f}$ 如下:

$$
M_{f} g=f g, \quad g \in W^{2,2}(\Omega) .
$$

设 $H$ 为可分的 Hilbert 空间, 记 $L(H)$ 为 $H$ 上所有有界线性算子构成的代 数, 对 $T \in L(H)$, 记 $\sigma(T)$ 为算子 $T$ 的谱. 设 $\mathcal{K}(H)$ 为 $L(H)$ 中所有紧算子构成的 理想. 从 $L(H)$ 到 Calkin 代数 $\mathcal{A}(H)=L(H) / \mathcal{K}(H)$ 的商映射记为 $\pi$, 则 $T$ 的本性谱 $\sigma_{e}(T)$ 为 $\pi(T)$ 在 Calkin 代数 $\mathcal{A}(H)$ 中的谱, 即 $\sigma_{e}(T)=\sigma(\pi(T))$. 称 $\mathbb{C} \backslash \sigma_{e}(T)$ 为 $T$ 的 Fredholm 域, 记为 $\rho_{F}(T)$. 显然 $\sigma_{e}(T)=\sigma_{l e}(T) \cup \sigma_{r e}(T)$, 其中 $\sigma_{l e}(T)=\sigma_{l}(\pi(T))$, $\sigma_{r e}(T)=\sigma_{r}(\pi(T))$. 记 $T$ 的 Wolf 谱 $\sigma_{l r e}(T)=\sigma_{l e}(T) \cap \sigma_{r e}(T)$. 称 $T$ 为一个半 Fredholm 算子, 如果 $T$ 的像 $\operatorname{ran} T$ 是闭的且 $\operatorname{nul} T:=\operatorname{dimker} T$ 或 $n u l T^{*}=\operatorname{dimker} T^{*}$ 中至少有一个为有限的, 此时 $T$ 的指标定义为 $\operatorname{ind} T=\operatorname{nul} T-\operatorname{nul} T^{*}, T$ 的半 Fredholm 域 $\rho_{s-F}(T):=\{\lambda \in \mathbb{C}: \lambda-T$ 是半 Fredholm 算子 $\}$.

记 $W(\Omega):=\left\{M_{f}: f \in W^{2,2}(\Omega)\right\}$, 则 $W(\Omega)$ 是一个严格循环的算子代数, $e(s, t) \equiv 1$ 为其严格循环向量. 一个 Hilbert 空间 $H$ 上的算子代数 $\mathcal{A}$ 称为是严格 循环的, 如果存在一个向量 $e \in H$, 使得 $\mathcal{A} e:=\{A e: A \in \mathcal{A}\}=H$. 设 $\mathcal{A}^{a}\left(M_{z}\right)$ 是由极点在 $\bar{\Omega}$ 外的 $M_{z}$ 的有理函数生成的弱闭代数, 记 $R(\Omega):=\mathcal{A}^{a}\left(M_{z}\right) e$. 因为 $W(\Omega)$ 是严格循环的, 由文献 [7], 存在整数 $N, K>0$, 使得对任意 $f \in W^{2,2}(\Omega)$,

$$
N\|f\|_{W^{2,2}(\Omega)} \leqslant\left\|M_{f}\right\| \leqslant K\|f\|_{W^{2,2}(\Omega)},
$$

因此 $R(\Omega)$ 是全体极点在 $\bar{\Omega}$ 外的有理函数在 $W^{2,2}(\Omega)$ 中的闭包.

命题 1.1 (i) $\sigma\left(M_{z}(\Omega)\right)=\bar{\Omega}, \sigma_{e}\left(M_{z}(\Omega)\right)=\partial \Omega$, 对任意 $z_{0} \in \Omega, \operatorname{nul}\left(M_{z}(\Omega)-\right.$ $\left.z_{0}\right)=0$ 且 $\operatorname{ind}\left(M_{z}(\Omega)-z_{0}\right)=-1$.

(ii) 交换子代数 $\mathcal{A}^{\prime}\left(M_{z}(\Omega)\right)$ 的极大理想恒同于 $\bar{\Omega}$. 对任意 $z_{0} \in \bar{\Omega}$, 它所对应 的乘法线性泛函 $k_{z_{0}}^{*}$ 为 $k_{z_{0}}^{*}\left(M_{f}(\Omega)\right)=f\left(z_{0}\right)=\left\langle f, k_{z_{0}}\right\rangle$ 对某个 $k_{z_{0}} \in R(\Omega)$ 成立, 且 $k_{z_{0}} \in \operatorname{ker}\left(M_{z}(\Omega)-z_{0}\right)^{*}$. 
(iii) $\mathcal{A}^{\prime}\left(M_{z}(\Omega)\right)=\mathcal{A}^{a}\left(M_{z}(\Omega)\right)=\left\{M_{f}(\Omega\}: f \in R(\Omega)\right\}$, 其中 $M_{f}(\Omega)=$ $\left.M_{f}\right|_{R(\Omega)}$, 因此 $\mathcal{A}^{\prime}\left(M_{z}(\Omega)\right)$ 是严格循环的, $M_{z}(\Omega)$ 是有理严格循环的.

证 (i) 如果 $z_{0} \notin \bar{\Omega}$, 则 $\left(z_{0}-z\right)^{-1} \in R(\Omega)$, 容易看出

$$
M_{\left(z_{0}-z\right)^{-1}}(\Omega)=\left(z_{0}-M_{z}(\Omega)\right)^{-1}
$$

因此 $\sigma\left(M_{z}(\Omega)\right) \subset \bar{\Omega}$.

设 $z_{0} \in \bar{\Omega}$, 则对任意 $g \in R(\Omega)$, 函数 $\left(z_{0}-M_{z}(\Omega)\right) g=\left(z_{0}-z\right) g(z)$ 在 $z_{0}$ 取值 为零, 从而 $z_{0}-M_{z}(\Omega)$ 不是映上的, 即 $\sigma\left(M_{z}(\Omega)\right)=\bar{\Omega}$.

当 $z_{0} \in \Omega$ 时, 对任一 $f \in R(\Omega)$, 都存在正数 $\delta>0$ 及在 $\Gamma$ 解析的函数 $h$, 使 得

$$
f(z)-f\left(z_{0}\right)=\left(z-z_{0}\right) h(z), \quad z \in \Gamma,
$$

这里 $\Gamma:=\left\{z \in \mathbb{C}:\left|z-z_{0}\right|<\delta\right\} \subset \bar{\Gamma} \subset \Omega$. 记

$$
E:=\Omega \backslash \frac{1}{3} \bar{\Gamma}=\Omega \backslash\left\{z \in \mathbb{C}:\left|z-z_{0}\right| \leqslant \delta / 3\right\} .
$$

定义

$$
g(z)= \begin{cases}h(z), & z \in \Gamma, \\ f(z)\left(z-z_{0}\right)^{-1}, & z \in E,\end{cases}
$$

则 $f(z)-f\left(z_{0}\right)=\left(z-z_{0}\right) g(z)$ 对 $z \in \Omega$ 成立, 且 $g$ 在 $\Omega$ 内解析. 由于

$$
\int_{E}|g|^{2} d m \leqslant \frac{9}{\delta^{2}} \int_{E}\left|\left(z-z_{0}\right) g(z)\right|^{2} d m \leqslant \frac{9}{\delta^{2}}\left\|f-f_{0}\right\|_{W^{2,2}(\Omega)}^{2},
$$

因此 $g \in L^{2}(E)$, 且

$$
\frac{\partial\left[f(z)-f\left(z_{0}\right)\right]}{\partial s}=f^{\prime}(z)=g(z)+\left(z-z_{0}\right) g^{\prime}(z) \in L^{2}(E) .
$$

我们有 $\left(z-z_{0}\right) g^{\prime}(z) \in L^{2}(E)$, 这样

$$
\int_{E}\left|g^{\prime}(z)\right|^{2} d m \leqslant \frac{9}{\delta^{2}} \int_{E}\left|\left(z-z_{0}\right) g^{\prime}(z)\right|^{2} d m \leqslant \frac{9}{\delta^{2}}\left\|f-f_{0}\right\|_{W^{2,2}(\Omega)}^{2},
$$

即 $g^{\prime} \in L^{2}(E)$. 因为

$$
\frac{\partial^{2}\left[f(z)-f\left(z_{0}\right)\right]}{\partial s^{2}}=2 g^{\prime}+\left(z-z_{0}\right) g^{\prime \prime} \in L^{2}(E),
$$

所以有 $\left(z-z_{0}\right) g^{\prime \prime} \in L^{2}(E)$, 这样

$$
\int_{E}\left|g^{\prime \prime}(z)\right| d m \leqslant \frac{9}{\delta^{2}}\left\|f-f_{0}\right\|_{W^{2,2}(\Omega)}^{2},
$$

即 $g \in W^{2,2}(E)$.

当 $k=0,1,2$ 且 $z \in \frac{2}{3} \Gamma=\left\{z \in \mathbb{C}:\left|z-z_{0}\right|<2 \delta / 3\right\}$ 时, 由 Cauchy 公式,

$$
\left|g^{(k)}(z)\right|=\left|\frac{1}{2 \pi \mathrm{i}} \int_{|\xi|=\partial \Gamma} \frac{g(\xi)}{(\xi-z)^{k+1}} d \xi\right| \leqslant \frac{3^{k+1}}{\delta^{k}} \max _{\xi \in \partial \Gamma}|g(\xi)|,
$$

因此 $g, g^{\prime}, g^{\prime \prime} \in L^{2}\left(\frac{2}{3} \Gamma\right)$, 进而 $g \in W^{2,2}\left(\frac{2}{3} \Gamma\right)$. 又由 $g \in W^{2,2}(E)$, 可知 $g \in W^{2,2}(\Omega)$. 于是由 $f(z)=f\left(z_{0}\right)+\left(z-z_{0}\right) g(z)$ 得 $\operatorname{ran}\left(M_{z}(\Omega)-z_{0}\right)$ 的余维数为 1 . 容易证明 
$\operatorname{ker}\left(M_{z}(\Omega)-z_{0}\right)=\{0\}$, 因此

$$
z_{0} \in \rho_{s-F}\left(M_{z}(\Omega)\right), \quad \rho_{s-F}\left(M_{z}(\Omega)\right)=\Omega
$$

且

$$
\operatorname{ind}\left(M_{z}(\Omega)-z_{0}\right)=-1 .
$$

(ii) 记 $\mathcal{A}=\left\{M_{f}(\Omega): f \in R(\Omega)\right\}$, 则易证 $\mathcal{A}$ 是一个弱算子闭的代数且 $\mathcal{A} e=$ $R(\Omega)$, 即 $\mathcal{A}$ 是严格循环的. 由文献 [7], $\mathcal{A}$ 的共轭空间为

$$
\mathcal{A}^{*}=\left\{g^{*} \text { : 存在某个 } g \in R(\Omega) \text {, 使得 } g^{*}\left(M_{f}(\Omega)\right)=\left\langle M_{f}(\Omega) e, g\right\rangle\right\} \text {. }
$$

这样，当 $z_{0} \in \bar{\Omega}$ 时，存在 $k_{z_{0}} \in R(\Omega)$, 使得

$$
k_{z_{0}}^{*}\left(M_{f}(\Omega)\right)=\left\langle M_{f}(\Omega) e, k_{z_{0}}\right\rangle=\left\langle f, k_{z_{0}}\right\rangle .
$$

反过来, 如果 $\phi: \mathcal{A} \rightarrow \mathbb{C}$ 是一个乘法线性泛函, 则

$$
\phi\left(M_{z}(\Omega)\right)=z_{0} \in \sigma\left(M_{z}(\Omega)\right)=\bar{\Omega} .
$$

对于极点在 $\bar{\Omega}$ 外的有理函数 $r(z)$, 有

$$
\phi\left(r\left(M_{z}(\Omega)\right)\right)=r\left(z_{0}\right) .
$$

因为 $\left\{r\left(M_{z}(\Omega)\right)\right\}$ 在 $\mathcal{A}$ 中稠密, 所以对任意 $f \in R(\Omega)$, 有 $\phi\left(M_{f}(\Omega)\right)=f\left(z_{0}\right)$.

因为对任意 $g \in R(\Omega)$, 有

$$
\left\langle g,\left(M_{z}(\Omega)-z_{0}\right)^{*} k_{z_{0}}\right\rangle=\left\langle\left(z-z_{0}\right) g, k_{z_{0}}\right\rangle=0,
$$

所以 $k_{z_{0}} \in \operatorname{ker}\left(\left(M_{z}(\Omega)\right)-z_{0}\right)^{*}$.

(iii) 设 $T \in \mathcal{A}^{\prime}\left(M_{z}(\Omega)\right)$, 则对 $z_{0} \in \Omega$, 有

$$
M_{z}(\Omega)^{*} T^{*} k_{z_{0}}=T^{*} M_{z}^{*}(\Omega) k_{z_{0}}=\overline{z_{0}} T^{*} k_{z_{0}} .
$$

因为 $\operatorname{nul}\left(M_{z}(\Omega)-z_{0}\right)^{*}=1$, 故存在某个复数 $t\left(z_{0}\right)$, 使得 $T^{*} k_{z_{0}}=\overline{t\left(z_{0}\right)} k_{z_{0}}$. 这样对 $f \in R(\Omega)$ 和 $z \in \Omega$, 有

$$
(T f)(z)=\left\langle T f, k_{z}\right\rangle=\left\langle f, T^{*} k_{z}\right\rangle=t(z) f(z) .
$$

又因为 $t(z)=(T e)(z) \in R(\Omega)$, 所以 $T=M_{t}(\Omega)$.

综上所述, $\mathcal{A}^{\prime}\left(M_{z}(\Omega)\right)=\mathcal{A}=\mathcal{A}^{a}\left(M_{z}(\Omega)\right)$.

命题 1.2 设 $\Omega$ 为单连通的解析 Cauchy 域, 则所有多项式构成的集合在 $R(\Omega)$ 中稠密.

证 对任一给定的函数 $f \in R(\Omega)$ 和任意小的正数 $\varepsilon$, 取极点在 $\bar{\Omega}$ 外的有 理函数 $r(z)$, 使得 $\|f-r\|_{W^{2,2}(\Omega)}<\varepsilon$. 对此 $r$, 存在有界解析 Cauchy 域 $\Omega_{1}$, 使得 $\bar{\Omega}_{1} \supset \Omega_{1} \supset \bar{\Omega} \supset \Omega$, 且 $r(z)$ 的极点在 $\bar{\Omega}_{1}$ 外. 由 Mergelyan 定理 ${ }^{[8]}$, 存在多项式 $p_{n}$, 使得 $p_{n}(z)$ 在 $\bar{\Omega}_{1}$ 中一致收玫到 $r(z)$, 即 $p_{n}(z) \stackrel{u}{\rightarrow} r(z), z \in \bar{\Omega}_{1}$. 由 Cauchy 公式可得

$$
p_{n}^{\prime}(z) \stackrel{u}{\rightarrow} r^{\prime}(z), \quad p_{n}^{\prime \prime}(z) \stackrel{u}{\rightarrow} r^{\prime \prime}(z), \quad z \in \Omega .
$$

总之可得 $p_{n}(z)$ 在 $W^{2,2}(\Omega)$ 中收玫到 $r(z)$, 因此存在正整数 $N$, 使得 $\left\|p_{N}-r\right\|_{W^{2,2}(\Omega)}$ $<\varepsilon$, 所以 $\left\|p_{N}-f\right\|_{W^{2,2}(\Omega)}<2 \varepsilon$. 证毕. 
记单位圆盘 $D=\{z \in \mathbb{C}:|z|<1\}$, 我们称 $R(D)$ 为 Sobolev 圆盘代数. 为方 便计, 在不引起混淆的情形下, 使用符号 $M_{f}$ 表示 $R(D)$ 上符号为 $f \in R(D)$ 的 乘法算子.

命题 1.3 (i) Hilbert 空间 $R(D)$ 有一组规范正交基 $\left\{e_{n}\right\}_{n=0}^{\infty}$, 满足

$$
e_{n}=\beta_{n} z^{n}, \quad \beta_{n}=\left[\frac{n+1}{\left(3 n^{4}-n^{2}+2 n+1\right) \pi}\right]^{1 / 2}, \quad n=0,1, \cdots .
$$

(ii) Sobolev 圆盘代数 $R(D)$ 是一个函数 Hilbert 空间, 其再生核为

对 $z_{0} \in \bar{\Omega}$,

$$
k(u, v)=\sum_{n=0}^{\infty} \beta_{n}^{2} u^{n} \bar{v}^{n},
$$

$$
k_{z_{0}}=\sum_{n=0}^{\infty}\left(\beta_{n}^{2}{\overline{z_{0}}}^{n}\right) z^{n} .
$$

(iii) 对任意在 $D$ 内解析的函数 $f=\sum_{n=0}^{\infty} f_{n} z^{n}, f \in R(D)$ 的充分必要条件 是

$$
\sum_{n=0}^{\infty} \frac{\left|f_{n}\right|^{2}}{\beta_{n}^{2}}<+\infty
$$

当 $f \in R(D)$ 时, 其部分和 $S_{n}=\sum_{k=0}^{n} f_{k} z^{k}$ 在 $\bar{D}$ 内一致收玫到 $f(z)$;

(iv) 乘自变量算子 $M_{z}$ 是本性正常的加权移位算子. $M_{z} e_{n}=\alpha_{n} e_{n+1}, \alpha_{n}=$ $\beta_{n} / \beta_{n+1}(n=0,1, \cdots)$, 且 $\left\|M_{z}\right\|=\sqrt{98 / 15}$;

(v) 对 $f(z)=\sum_{n=0}^{\infty} f_{n} z^{n} \in R(D)$, 乘法算子 $M_{f}$ 可表示为

$$
M_{f}=\left(\begin{array}{cccccc}
f_{0} & & & & \\
f_{1} \frac{\beta_{0}}{\beta_{1}} & f_{0} & & & \\
f_{2} \frac{\beta_{0}}{\beta_{2}} & f_{1} \frac{\beta_{1}}{\beta_{2}} & f_{0} & & \\
f_{3} \frac{\beta_{0}}{\beta_{3}} & f_{2} \frac{\beta_{1}}{\beta_{3}} & f_{1} \frac{\beta_{2}}{\beta_{3}} & f_{0} & \\
\vdots & \vdots & \ldots & & \ddots
\end{array}\right) \begin{gathered}
e_{0} \\
e_{1} \\
e_{2} . \\
e_{3} \\
\vdots
\end{gathered}
$$

证 (i) 由计算可知 $z^{n}=(s+\mathrm{i} t)^{n}(n=0,1, \cdots)$ 为一组正交系, 且

$$
\left\|z^{n}\right\|^{2}=\frac{\left(3 n^{4}-n^{2}+2 n+1\right) \pi}{n+1} .
$$

由命题 $1.2,\left\{e_{n}\right\}_{n=0}^{\infty}$ 构成 $R(D)$ 的一组规范正交基.

(ii) 对任一 $z_{0} \in \bar{D}$ 和 $f \in R(D),\left|f\left(z_{0}\right)\right| \leqslant\|f\|_{C(\bar{\Omega})} \leqslant M\|f\|_{W_{2,2}(\Omega)}$, 因此 $R(D)$ 是一个具有再生核的函数 Hilbert 空间, 其再生核为

$$
k(u, v)=\sum_{n=0}^{\infty} e_{n}(u) \overline{e_{n}(v)}=\sum_{n=0}^{\infty} \beta_{n}^{2} u^{n} \bar{v}^{n} .
$$

由于

$$
\left\langle f, \sum_{n=0}^{\infty} \beta_{n}^{2}{\overline{z_{0}}}^{n} z^{n}\right\rangle=f\left(z_{0}\right)
$$

www.scichina.com 
命题 1.1 中的 $k_{z_{0}}$ 可表示为

$$
k_{z_{0}}=\sum_{n=0}^{\infty}\left(\beta_{n}^{2}{\overline{z_{0}}}^{n}\right) z^{n} .
$$

(iii) 设 $f$ 在 $D$ 内解析, 其 Taylor 展式为 $f=\sum_{n=0}^{\infty} f_{n} z^{n}$, 则

$$
f=\sum_{n=0}^{\infty} \frac{f_{n}}{\beta_{n}} e_{n} \in R(D)
$$

当且仅当

$$
\sum_{n=0}^{\infty}\left|\frac{f_{n}}{\beta_{n}}\right|^{2}<+\infty
$$

当 $f \in R(D)$ 时,

$$
S_{n}(z)=\sum_{k=0}^{n} f_{k} z^{k}=\sum_{k=0}^{n} \frac{f_{k}}{\beta_{k}} e_{k} \stackrel{W^{2,2}(\Omega)}{\longrightarrow} f \quad(n \rightarrow \infty) .
$$

由 Sobolev 嵌入定理, $S_{n}(z) \stackrel{u}{\rightarrow} f(z), z \in \bar{D}$.

(iv) 由于 $M_{z} e_{n}=z \beta_{n} z^{n}=\frac{\beta_{n}}{\beta_{n+1}} e_{n+1}=\alpha_{n} e_{n+1}(n=0,1,2, \cdots)$, 故

$$
M_{z}^{*} M_{z}-M_{z} M_{z}^{*}=\left(\begin{array}{llll}
\alpha_{0}^{2} & & & 0 \\
& \alpha_{1}^{2}-\alpha_{0}^{2} & & \\
& & \alpha_{2}^{2}-\alpha_{1}^{2} & \\
0 & & & \ddots
\end{array}\right) \text {, }
$$

注意到 $\alpha_{n+1}^{2}-\alpha_{n}^{2} \rightarrow 0$, 所以 $M_{z}$ 是紧的.

(v) 设 $f=\sum_{n=0}^{\infty} f_{n} z^{n}=\sum_{n=0}^{\infty} \frac{f_{n}}{\beta_{n}} e_{n} \in R(D)$, 则

$$
\begin{aligned}
\left\langle M_{f} e_{n}, e_{m}\right\rangle & =\left\langle\sum_{k=0}^{\infty} f_{k} z^{k} \beta_{n} z^{n}, e_{m}\right\rangle \\
& =\left\langle\sum_{k=0}^{\infty} f_{k} \frac{\beta_{n}}{\beta_{n+k}} e^{n+k}, e_{m}\right\rangle= \begin{cases}f_{m-n} \frac{\beta_{n}}{\beta_{m}}, & m \geqslant n, \\
0, & m<n .\end{cases}
\end{aligned}
$$

所以 $M_{f}$ 可表示为 (v) 中的矩阵. 证毕.

设 $f \in R(D)$, 我们记 $f_{r}:=f(r z), z \in \bar{D}, 0<r \leqslant 1$.

命题 1.4 (i) 当 $r \rightarrow 1$ 时, $f_{r}$ 在 $R(D)$ 中收玫到 $f$;

(ii) 当 $r \rightarrow 1$ 时, $f_{r}\left(M_{z}\right)$ 按算子范数收敛到 $M_{f}$.

证 (i) 因为 $f(z)$ 在 $\bar{D}$ 上一致连续, $f(r z)$ 在 $\bar{D}$ 上一致收玫到 $f(z)$, 因此

$$
\int_{D}|f(r z)-f(z)|^{2} d m \rightarrow 0 \quad(r \rightarrow 1) .
$$

对任意的正数 $\varepsilon$, 因为 $f^{\prime}, f^{\prime \prime} \in L^{2}(D)$, 所以可以选定 $r_{0}\left(0<r_{0}<1\right)$, 使得

$$
\int_{D \backslash\left(2 r_{0}-1\right) D}\left|f^{\prime}(z)\right|^{2} d m<\varepsilon, \quad \int_{D \backslash\left(2 r_{0}-1\right) D}\left|f^{\prime \prime}(z)\right|^{2} d m<\varepsilon .
$$


这时有

$$
\begin{aligned}
\int_{D}\left|r f^{\prime}(r z)-f^{\prime}(z)\right|^{2} d m= & \int_{D \backslash r_{0} D}\left|r f^{\prime}(r z)-f^{\prime}(z)\right|^{2} d m+\int_{r_{0} D}\left|r f^{\prime}(r z)-f^{\prime}(z)\right|^{2} d m \\
\leqslant & 2\left[\int_{D \backslash r_{0} D}\left|r f^{\prime}(r z)\right|^{2} d m+\int_{D \backslash r_{0} D}\left|f^{\prime}(z)\right|^{2} d m\right] \\
& +\int_{r_{0} D}\left|r f^{\prime}(r z)-f^{\prime}(z)\right|^{2} d m .
\end{aligned}
$$

类似地, 有

$$
\begin{aligned}
\int_{D}\left|r^{2} f^{\prime \prime}(r z)-f^{\prime \prime}(z)\right|^{2} d m \leqslant & {\left[\int_{D \backslash r_{0} D}\left|r^{2} f^{\prime \prime}(r z)\right|^{2} d m+\int_{D \backslash r_{0} D}\left|f^{\prime \prime}(z)\right|^{2} d m\right] } \\
& +\int_{r_{0} D}\left|r^{2} f^{\prime \prime}(r z)-f^{\prime \prime}(z)\right|^{2} d m .
\end{aligned}
$$

因为 $r f^{\prime}(r z)$ 和 $r^{2} f^{\prime \prime}(r z)$ 在 $D$ 内分别内闭一致收玫到 $f^{\prime}(z)$ 和 $f^{\prime \prime}(z)$, 所以可以选 择 $r_{1}>0$, 使得当 $r>r_{1}$ 时, 有

(a) 当 $|z| \leqslant r_{0}$ 时, $\left|r f^{\prime}(r z)-f^{\prime}(z)\right|<\varepsilon$ 且 $\left|r^{2} f^{\prime \prime}(r z)-f^{\prime \prime}(z)\right|<\varepsilon$;

(b) 当 $z \in D \backslash r_{0} D$ 时, $r z>2 r_{0}-1$.

这样, 当 $r>r_{1}$ 时, 我们有

$$
\int_{D}\left|r f^{\prime}(r z)-f^{\prime}(z)\right|^{2} d m<4 \varepsilon+\pi \varepsilon^{2}
$$

和

$$
\int_{D}\left|r^{2} f^{\prime \prime}(r z)-f^{\prime \prime}(z)\right|^{2} d m<4 \varepsilon+\pi \varepsilon^{2},
$$

即 $f_{r} \stackrel{R(D)}{\longrightarrow} f(r \rightarrow 1)$.

(ii) 对任意 $g \in R(D)$,

$$
\begin{aligned}
f_{r}\left(M_{z}\right) g & =\frac{1}{2 \pi \mathrm{i}} \int_{|\xi|=1 / r} f(r \xi)\left(\xi-M_{z}\right)^{-1} g(z) d \xi \\
& =\frac{1}{2 \pi \mathrm{i}} \int_{|\xi|=1 / r} f(r \xi)(\xi-z)^{-1} g(z) d \xi=f(r z) g(z) \quad(z \in \bar{D}) .
\end{aligned}
$$

如果 $f(z)=\sum_{n=0}^{\infty} f_{n} z^{n}$, 则 $f_{r}(z)=\sum_{n=0}^{\infty} f_{n} r^{n} z^{n}$. 因为

$$
\sum_{n=0}^{\infty}\left|\frac{f_{n} r^{n}}{\beta_{n}}\right|^{2} \leqslant \sum_{n=0}^{\infty}\left|\frac{f_{n}}{\beta_{n}}\right|^{2}<+\infty,
$$

由命题 1.3, $f_{r} \in R(D)$, 于是 $f_{r}\left(M_{z}\right)=M_{f_{r}}$. 因为 $\mathcal{A}^{\prime}\left(M_{z}\right)$ 是严格循环的, $f_{r}$ 在 $R(D)$ 中收玫到 $f$ 等价于 $f_{r}\left(M_{z}\right)$ 收玫到 $M_{f}$, 即 $M_{f_{r}}$ 收玫到 $M_{f}$.

定理 1.5 设 $f \in R(D)$, 则

(i) $\sigma\left(M_{f}\right)=f(\bar{D})$.

(ii) $\sigma_{e}\left(M_{f}\right)=\sigma_{\text {lre }}\left(M_{f}\right)=f(\mathbb{T})$, 其中 $\mathbb{T}=\partial D$. 如果 $z_{0} \in D, f\left(z_{0}\right) \notin f(\mathbb{T})$, 则 $\operatorname{ind}\left(M_{f}-f\left(z_{0}\right)\right)=-\operatorname{nul}\left(M_{f}-f\left(z_{0}\right)\right)^{*}=-n$,

这里 $n$ 为 $f(z)-f\left(z_{0}\right)$ 在 $D$ 内的零点的个数 (包含重数). 
证 (i) 当 $z_{0} \in \bar{D}$ 时, $M_{f}-f\left(z_{0}\right)$ 的值域中的函数在 $z_{0}$ 点取值为零, 所以 $M_{f}-f\left(z_{0}\right)$ 不是映上的, 从而 $\sigma\left(M_{f}\right) \supset f(\bar{D})$. 另一方面, 如果 $w_{0} \notin f(\bar{D})$, 则易证

$$
\left(f(z)-w_{0}\right)^{-1} \in R(D),
$$

因此

$$
\left(M_{f}-w_{0}\right) M_{\left(f-w_{0}\right)^{-1}}=M_{\left(f-w_{0}\right)^{-1}}\left(M_{f}-w_{0}\right)=I,
$$

于是 $\sigma\left(M_{f}\right)=f(\bar{D})$.

(ii) 由命题 1.1, $\sigma_{e}\left(M_{z}\right)=\sigma_{l r e}\left(M_{z}\right)=\mathbb{T}$. 由文献 [9],

$$
\sigma_{e}\left(M_{f_{r}}\right)=\sigma_{\text {lre }}\left(M_{f_{r}}\right)=\sigma_{e}\left(f_{r}\left(M_{z}\right)\right)=f_{r}(\mathbb{T})=\{f(z):|z|=r\},
$$

因此如果 $z_{0} \in \mathbb{T}$, 则 $f\left(r z_{0}\right) \in \sigma_{l r e}\left(M_{f_{r}}\right)$. 因为

$$
M_{f_{r}}-f\left(r z_{0}\right) \rightarrow M_{f}-f\left(z_{0}\right) \quad(r \rightarrow 1),
$$

所以 $f\left(z_{0}\right) \in \sigma_{\text {lre }}\left(M_{f}\right)$. 这说明 $f(\mathbb{T}) \subset \sigma_{\text {lre }}\left(M_{f}\right)$.

若存在 $z_{0} \in D$, 使得 $f\left(z_{0}\right) \notin f(\mathbb{T})$, 则 $f(z)-f\left(z_{0}\right)$ 在 $\bar{D}$ 内只有有限多个零 点, 不妨设之为 $\left\{z_{0}, z_{1}, \cdots, z_{n}\right\} \subset D$. 由命题 1.1(i) 的证明可知

$$
f(z)-f\left(z_{0}\right)=\left(z-z_{0}\right)^{k_{0}}\left(z-z_{1}\right)^{k_{1}} \cdots\left(z-z_{n}\right)^{k_{n}} g(z),
$$

其中 $g \in R(D)$ 且 $g(z) \neq 0, z \in \bar{D}$.

由命题 1.1,

$$
M_{f}-f\left(z_{0}\right)=\left(M_{z-z_{0}}\right)^{k_{0}} \cdots\left(M_{z-z_{n}}\right)^{k_{n}} M_{g}
$$

为 Fredholm 算子, 且

$$
\operatorname{ind}\left(M_{f}-f\left(z_{0}\right)=-\sum_{i=0}^{n} k_{i}=n .\right.
$$

显然 $\operatorname{nul}\left(M_{f}-f\left(z_{0}\right)\right)=0$, 因此 $\operatorname{nul}\left(M_{f}-f\left(z_{0}\right)\right)^{*}=n$. 证毕.

设 $H$ 是一个 Hilbert 空间, $\Omega$ 是 $\mathbb{C}$ 中的有界开集， $n$ 为一自然数，记 $B_{n}(\Omega)$ 为 $H$ 上满足下列条件的有界线性算子 $B$ 的全体 ${ }^{[10]}$ :

(i) $\sigma(B) \supset \Omega$;

(ii) $\operatorname{ran}(B-\lambda)=H$, 对任意 $\lambda \in \Omega$;

(iii) $\operatorname{nul}(B-\lambda)=n$, 对任意 $\lambda \in \Omega$;

(iv) $\vee\{\operatorname{ker}(B-\lambda): \lambda \in \Omega\}=H$,

其中 (iv) 可由下面的等价条件 (iv) ${ }^{\prime}$ 代替 (见文献 [5]):

(iv) $)^{\prime}$ 存在 $\lambda_{0} \in \Omega$, 使得 $\vee\left\{\operatorname{ker}\left(B-\lambda_{0}\right)^{k}: k \geqslant 1\right\}=H$.

定理 1.6 设 $f \in R(D), z_{0} \in D, f\left(z_{0}\right) \notin f(\mathbb{T})$. 记 $M_{f}$ 的半 Fredholm 域 $\rho_{s-F}\left(M_{f}\right)$ 中包含 $f\left(z_{0}\right)$ 的区域为 $\Omega$, 则 $M_{f}^{*} \in B_{n}(\Omega)$, 其中 $n$ 为函数 $f(z)-f\left(z_{0}\right)$ 在 $D$ 内零点的个数.

证 只需证明 $\vee\left\{\operatorname{ker}\left[\left(M_{f}-f\left(z_{0}\right)\right)^{*}\right]^{k}: k \geqslant 1\right\}=R(D)$. 假设

$$
y \in R(D) \ominus \vee\left\{\operatorname{ker}\left[\left(M_{f}-f\left(z_{0}\right)\right)^{*}\right]^{k}: k \geqslant 1\right\},
$$


则对任意 $k$, 有

$$
y \in\left[\operatorname{ker}\left(M_{f}-f\left(z_{0}\right)\right)^{* k}\right]^{\perp}=\operatorname{ran}\left(M_{f}-f\left(z_{0}\right)\right)^{k},
$$

因此我们可以找到一个函数 $h \in R(D)$, 使得

$$
y=\left[M_{f}-f\left(z_{0}\right)\right]^{k} h=\left(z-z_{0}\right)^{k_{0} k} \cdots\left(z-z_{n}\right)^{k_{n} k} g^{k} h .
$$

注意到 $z_{0}$ 是 $y$ 的 $k k_{0}$ 阶零点, 而 $k \geqslant 1$ 且 $k$ 遍历所有正整数, 所以 $z_{0}$ 是 $y$ 的本 性奇点, 得出矛盾.

例 1.7 设 $f(z)=2 z^{2}-z$, 则 $f(\mathbb{T})$ 把 $f(\bar{D})$ 分成 $\Omega_{1}$ 和 $\Omega_{2}$ 两部分, 其中 $0 \in \Omega_{1}, 2 \in \Omega_{2}$, 并且 $M_{f}^{*} \in B_{2}\left(\Omega_{1}\right)$, 同时 $M_{f}^{*} \in B_{1}\left(\Omega_{2}\right)$.

在与蒋春澜的交流中, 他给出了如下命题的证明:

命题 1.8 对任意 $f \in R(D), M_{f}$ 是本性正常的.

证 由命题 1.2, 存在一列多项式 $p_{n}$, 使得 $p_{n}$ 在 $R(D)$ 中收玫到 $f$. 因为 $\mathcal{A}^{\prime}\left(M_{z}\right)=\left\{M_{g}: g \in R(D)\right\}$ 是一个严格循环的算子代数, 从而 $M_{p_{n}} \rightarrow M_{f}(n \rightarrow$ $\infty)$. 命题 1.3 (iv) 中已证明 $M_{z}$ 是本性正常的, 故 $\pi\left(M_{z}\right)$ 在 Calkin 代数 $\mathcal{A}(R(D))$ 中是正常的, 从而 $\pi\left(M_{p_{n}}\right)$ 是正常的, 由此可知 $\pi\left(M_{f}\right)=\lim _{n \rightarrow \infty} \pi\left(M_{p_{n}}\right)$ 也是正常 的, 即 $M_{f}$ 是 $L(R(D))$ 中的本性正常算子.

\section{2 乘法算子 $M_{f}$ 的换位子}

命题 2.1 设 $f \in R(D), M_{f}^{*} \in B_{n}(\Omega), z_{0} \in D_{1}:=f^{-1}(\Omega)$, 且

$$
f(z)-f\left(z_{0}\right)=\left(z-z_{0}\right)^{h_{1}}\left(z-z_{1}\right)^{h_{2}} \cdots\left(z-z_{l}\right)^{h_{l+1}} g_{z_{0}}(z),
$$

其中 $\left\{z_{i}\right\}_{i=1}^{l} \subset D_{1}$ 互不相同, $\sum_{i=1}^{l+1} h_{i}=n, g_{z_{0}}(z) \neq 0, z \in \bar{D}$, 则存在 $n$ 个线性无 关向量

$$
K_{z_{0}}:=\left\{k_{z_{0}}, k_{z_{0}}^{1}, \cdots, k_{z_{0}}^{h_{1}-1}, k_{z_{1}}, \cdots, k_{z_{1}}^{h_{2}-1}, \cdots, k_{z_{l}}^{h_{l+1}-1}\right\},
$$

使得 $\operatorname{ker} M_{f-f\left(z_{0}\right)}^{*}=\vee K_{z_{0}}$.

证 取 $k_{z_{0}}^{1}, k_{z_{0}}^{2}, \cdots, k_{z_{0}}^{h_{1}-1} \in R(D)$, 使之满足 $M_{z-z_{0}}^{*} k_{z_{0}}^{1}=k_{z_{0}}, M_{z-z_{0}}^{*} k_{z_{0}}^{2}=$ $k_{z_{0}}^{1}, \cdots, M_{z-z_{0}}^{*} k_{z_{0}}^{h_{1}-1}=k_{z_{0}}^{h_{1}-2}$. 令 $k_{z_{0}}^{0}=k_{z_{0}}$, 则对任意 $u \in R(D), 0 \leqslant j \leqslant h_{1}-1$,

$$
\begin{aligned}
\left\langle u, M_{f-f\left(z_{0}\right)}^{*} k_{z_{0}}^{j}\right\rangle & =\left\langle\left(f-f\left(z_{0}\right)\right) u, k_{z_{0}}^{j}\right\rangle \\
& =\left\langle\left(z-z_{0}\right)^{j}\left(z-z_{0}\right)^{h_{1}-j}\left(z-z_{1}\right)^{h_{2}} \cdots\left(z-z_{l}\right)^{h_{l+1}} g_{z_{0}}(z) u(z), k_{z_{0}}^{j}\right\rangle \\
& =\left\langle\left(z-z_{0}\right)^{h_{1}-j}\left(z-z_{1}\right)^{h_{2}} \cdots\left(z-z_{l}\right)^{h_{l+1}} g_{z_{0}} u, k_{z_{0}}\right\rangle=0,
\end{aligned}
$$

因此 $k_{z_{0}}^{j} \in \operatorname{ker} M_{f-f\left(z_{0}\right)}^{*}$. 类似地, 取 $k_{z_{i}}^{1}, \cdots, k_{z_{i}}^{h_{i+1}-1} \in R(D)$, 使得

$$
M_{z-z_{i}}^{*} k_{z_{i}}^{1}=k_{z_{i}}, \cdots, M_{z-z_{i}}^{*} k_{z_{i}}^{h_{i+1}-1}=k_{z_{i}}^{h_{i+1}-2} \quad(0<i \leqslant l),
$$

则

$$
k_{z_{i}}^{j} \in \operatorname{ker} M_{f-f\left(z_{0}\right)}^{*} \quad\left(0 \leqslant j \leqslant h_{i+1}-1\right) .
$$

如果存在复数序列 $\left\{c_{i}^{j} ; 0 \leqslant i \leqslant l, 0 \leqslant j \leqslant h_{i}-1\right\}$ 满足 $\sum_{i, j} c_{i}^{j} k_{z_{i}}^{j}=0$, 则在上式

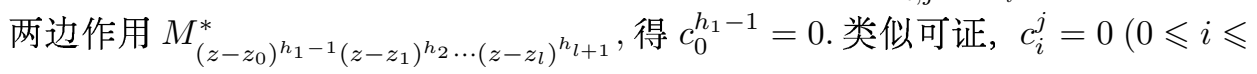


$\left.l, 0 \leqslant j \leqslant h_{i}-1\right)$, 即 $K_{z_{0}}$ 是线性无关的, 因此 $\operatorname{ker} M_{f-f\left(z_{0}\right)}^{*}=\vee K_{z_{0}}$. 计算表明, 特别可取

$$
\begin{aligned}
k_{z_{0}}^{1}(z) & \left.=\sum_{n=1}^{\infty} n \beta_{n}^{2}{\overline{z_{0}}}^{n-1} z^{n}=\overline{\left[\frac{d \bar{k}_{u}}{d u}\right]}\right]_{u=z_{0}}, \\
k_{z_{0}}^{2}(z) & =\frac{1}{2} \sum_{n=2}^{\infty} n(n-1) \beta_{n}^{2}{\overline{z_{0}}}^{n-2} z^{n}=\frac{1}{2} \overline{\left[\frac{d^{2} \bar{k}_{u}}{d^{2} u}\right]_{u=z_{0}}} \\
& \cdots, \\
k_{z_{0}}^{j}(z) & =\frac{1}{j !} \sum_{n=j}^{\infty} n(n-1) \cdots(n-j+1) \beta_{n}^{2}{\overline{z_{0}}}^{n-j} z^{n}=\overline{\left[\frac{d^{j} \bar{k}_{u}}{d^{j} u}\right]}
\end{aligned}
$$

类似可取 $k_{z_{i}}^{j}$. 证毕.

对 $f \in R(D)$, 设 $M_{f}^{*} \in B_{n}(\Omega), D_{1}=f^{-1}(\Omega)$, 则显然对 $z_{0} \in D_{1}$, 有

$$
f(z)-f\left(z_{0}\right)=\left(z-z_{0}\right)\left(z-z_{1}\right) \cdots\left(z-z_{n-1}\right) g_{z_{0}}(z), \quad g_{z_{0}}(z) \neq 0, \quad z \in \bar{D} .
$$

定理 2.2 设 $f \in R(D), M_{f}^{*} \in B_{n}(\Omega)$, 则存在区域 $\Lambda \subset f^{-1}(\Omega)$ 及 $\Lambda$ 上的解 析函数 $\alpha_{1}(z), \cdots, \alpha_{n}(z)$ 和 $Z_{1}(z), \cdots, Z_{n-1}(z)$, 使得对任意 $A \in \mathcal{A}^{\prime}\left(M_{f}\right)$, 有

$$
(A g)(z)=\alpha_{1}(z) g(z)+\alpha_{2}(z) g\left(Z_{1}(z)\right)+\cdots+\alpha_{n}(z) g\left(Z_{n-1}(z)\right),
$$

其中 $g \in R(D), z \in \Lambda$.

证 由于 $f(z)$ 在 $D_{1}=f^{-1}(\Omega)$ 内解析, 所以在 $D$ 内使得 $f^{\prime}(z)=0$ 的点至 多有可数个, 从而可以选取它的一个内闭子集 $D_{2}$, 使得 $\left\{z \in D_{2}: f^{\prime}(z)=0\right\}$ 是一 个有限集, 令 $\Lambda=\stackrel{\circ}{D}_{2}$, 即 $D_{2}$ 的内部. 特别当 $\Gamma=\left\{z \in D_{1}: f^{\prime}(z)=0\right\}$ 为有限集 时, 可取 $\Lambda=D_{1} \backslash \Gamma$. 对 $z \in \Lambda$, 由命题 2.1, $\operatorname{ker} M_{f-f(z)}^{*}=\bigvee\left\{k_{z}, k_{z_{1}}, \cdots, k_{z_{n-1}}\right\}$, 记 $N_{z}=\left\{z, z_{1}, \cdots, z_{n-1}\right\}$, 则 $N_{z}$ 中的点两两不同. 由于 $A \in \mathcal{A}^{\prime}\left(M_{f}\right)$, 所以 $A^{*} k_{z} \in$ $\operatorname{ker} M_{f-f(z)}^{*}$, 因此存在复数 $\overline{\alpha_{1}(z)}, \overline{\alpha_{2}(z)}, \cdots, \overline{\alpha_{n}(z)}$, 使得

$$
A^{*} k_{z}=\overline{\alpha_{1}(z)} k_{z}+\overline{\alpha_{2}(z)} k_{z_{1}}+\cdots+\overline{\alpha_{n}(z)} k_{z_{n-1}},
$$

从而有

$$
(A g)(z)=\left\langle A g, k_{z}\right\rangle=\left\langle g, A^{*} k_{z}\right\rangle=\alpha_{1}(z) g(z)+\alpha_{2}(z) g\left(z_{1}\right)+\cdots+\alpha_{n}(z) g\left(z_{n-1}\right) .
$$

对 $N_{z}=\left\{z, z_{1}, \cdots, z_{n-1}\right\}$, 选择 $u_{1}, u_{2}, \cdots, u_{n-1} \in D_{1}$, 使得

(i) 对某个 $\varepsilon>0, n$ 个开球 $B(z, \varepsilon), B\left(z_{k}, \varepsilon\right)(1 \leqslant k \leqslant n-1)$ 在 $D_{1}$ 内且两两 互不相交;

(ii) $\left\{u_{k}\right\}_{k=1}^{n-1}$ 两两不等且 $\left\{u_{k}\right\}_{k=1}^{n-1} \cap\left[\bar{B}(z, \varepsilon) \cup\left[\cup_{k=1}^{n-1} \bar{B}\left(z_{k}, \varepsilon\right)\right]\right]=\emptyset$.

令

$$
f_{k}\left(Z, Z_{1}, \cdots, Z_{n-1}\right)=\left(u_{k}-Z\right)\left(u_{k}-Z_{1}\right) \cdots\left(u_{k}-Z_{n-1}\right) g_{z}\left(u_{k}\right)-f\left(u_{k}\right)+f(Z),
$$


其中 $Z, Z_{1}, \cdots, Z_{n-1} \in D_{1} \backslash \Gamma, k=1,2, \cdots, n-1$, 满足 (1) 式. 计算表明

$$
\begin{aligned}
|\Delta| & =\left|\operatorname{det}\left[\left.\frac{\partial f_{k}}{\partial Z_{i}}\right|_{\left(Z, Z_{1}, \cdots, Z_{n-1}\right)=\left(z, z_{1}, \cdots, z_{n-1}\right)}\right]\right| \\
& =\left|\left[\prod_{i \neq j}\left(u_{i}-u_{j}\right)\right]\left[\prod_{i \neq j}\left(z_{i}-z_{j}\right)\right]\left[\prod_{i=1}^{n-1}\left(u_{i}-z\right)\right]\left[\prod_{i=1}^{n-1} g_{z}\left(u_{i}\right)\right]\right| \\
& \neq 0 .
\end{aligned}
$$

由全纯函数的隐函数定理 (如文献 [11] 定理 9.6), 存在 $\delta>0$ 和 $B(z, \delta)$ 中的解析 函数 $Z_{k}=Z_{k}(v)(k=1,2, \cdots, n-1)$, 对任意 $v \in B(z, \delta), k=1,2, \cdots, n-1$,

$$
f\left(u_{k}\right)-f(v)=\left(u_{k}-v\right)\left(u_{k}-Z_{1}(v)\right) \cdots\left(u_{k}-Z_{n-1}(v)\right) g_{v}\left(u_{k}\right) .
$$

这样, 对 $v \in B(z, \delta), v, Z_{1}(v), \cdots, Z_{n-1}(v)$ 满足 (1) 式, 且解析函数 $Z_{1}(v), \cdots$, $Z_{n-1}(v)$ 可延拓到 $\Lambda$, 并满足

$$
f(u)-f(z)=(u-z)\left(u-Z_{1}(z)\right) \cdots\left(u-Z_{n-1}(z)\right) g_{z}(u) .
$$

这样, (3) 式变为

$(A g)(z)=\alpha_{1}(z) g(z)+\alpha_{2}(z) g\left(Z_{1}(z)\right)+\cdots+\alpha_{n}(z) g\left(Z_{n-1}(z)\right), \quad \forall g \in R(D), z \in \Lambda$. 分别取 $g=e, z, z^{2}, \cdots, z^{n-1}$, 并记 $h_{1}=A e, h_{2}=A z, \cdots, h_{n}=A z^{n-1}$, 则

$$
\left\{\begin{array}{l}
\alpha_{1}(z)+\alpha_{2}(z)+\cdots+\alpha_{n}(z)=h_{1}(z) \\
z \alpha_{1}(z)+Z_{1}(z) \alpha_{2}(z)+\cdots+Z_{n-1}(z) \alpha_{n}(z)=h_{2}(z) \\
\cdots \\
z^{n-1}(z) \alpha_{1}(z)+Z_{1}^{n-1}(z) \alpha_{2}(z)+\cdots+Z_{n-1}^{n-1}(z) \alpha_{n}(z)=h_{n}(z) .
\end{array}\right.
$$

注意到系数行列式 $J(z)$ 是一个 Vandermonde 行列式,

$$
J(z)=\left|\begin{array}{cccc}
1 & 1 & \ldots & 1 \\
z & Z_{1} & \ldots & Z_{n-1} \\
z^{2} & Z_{1}^{2} & \ldots & Z_{n-1}^{2} \\
\vdots & \vdots & & \vdots \\
z^{n-1} & Z_{1}^{n-1} & \ldots & Z_{n-1}^{n-1}
\end{array}\right| \neq 0
$$

由 Cramer 法则, $\alpha_{1}(z), \cdots, \alpha_{n}(z)$ 在 $D_{1} \backslash \Gamma$ 上解析. 因为 $A g$ 是解析函数, 所以 (3) 式完全确定了 $A g$, 也完全刻画了 $A \in \mathcal{A}^{\prime}\left(M_{f}\right)$.

推论 2.3 若 $M_{f}^{*} \in B_{1}(\Omega)$, 则 $\mathcal{A}^{\prime}\left(M_{f}\right)=\left\{M_{g}: g \in R(D)\right\}$.

推论 2.3 指出, 如果 $M_{f}^{*} \in B_{1}(\Omega)$, 则 $\mathcal{A}^{\prime}\left(M_{f}\right)$ 是可交换的, 其实这是 $M_{f}^{*}$ 为 $B_{1}(\Omega)$ 算子的特征. 在进一步讨论前需要下面的概念和引理:

定义 2.4 设 $T \in B_{n}(\Omega)$, 正整数 $n$ 称为是 $T$ 的极小指标, 如果存在正整数 $m$, 使得 $T \in B_{m}\left(\Omega_{1}\right)$ 对某开集 $\Omega_{1}$ 成立，则 $n \leqslant m$ (见文献 [12]). 
对 $A \in \mathcal{A}^{\prime}(T)$ 及 $u \in \Omega$, 记 $A(u):=\left.A\right|_{\operatorname{ker}(T-u)}$.

引理 2.5 (文献 [12], 定理 2.8) 设 $T$ 是一个强不可约算子, $T \in B_{n}(\Omega)$ 且具 有极小指标 $n$, 则对任意 $u \in \Omega, \sigma(A(u))$ 是连通的.

一个算子 $T$ 称为强不可约的, 如果对任意可逆算子 $X, X T X^{-1}$ 都是不可约 的.

定理 2.6 设 $f \in R(D)$, 则下列结果等价:

(i) $M_{f}^{*} \in B_{1}(\Omega)$;

(ii) $\mathcal{A}^{\prime}\left(M_{f}\right)=\left\{M_{g}: g \in R(D)\right\}$;

(iii) $\mathcal{A}^{\prime}\left(M_{f}\right)$ 交换;

(iv) 如果 $M_{f}^{*} \in B_{n}\left(\Omega_{1}\right)$, 则对任意 $A \in \mathcal{A}^{\prime}\left(M_{f}\right)$,

$$
(A z)(z)=z h_{1}(z), \quad\left(A z^{2}\right)(z)=z^{2} h_{1}(z), \cdots,\left(A z^{n-1}\right)(z)=z^{n-1} h_{1}(z),
$$

其中 $h_{1}=A e$;

(v) $M_{f}$ 是强不可约的.

证 (i) $\Rightarrow$ (ii). 推论 2.3.

(ii) $\Rightarrow$ (iii). 显然.

(iii) $\Rightarrow$ (iv). 若不然, 假设存在算子 $A \in \mathcal{A}^{\prime}\left(M_{f}\right)$, 使得 $\left(A z^{k}\right)\left(z_{0}\right) \neq z^{k} h_{1}\left(z_{0}\right)$ 对某个 $k(1<k \leqslant n-1)$ 及 $z_{0} \in D_{1}\left(z_{0} \neq 0\right)$ 成立, 则

$$
\left(M_{z^{k}} A \cdot e\right)\left(z_{0}\right)=z_{0}^{k} h_{1}\left(z_{0}\right) \neq\left(A z^{k}\right)\left(z_{0}\right)=\left(A M_{z^{k}} \cdot e\right)\left(z_{0}\right),
$$

因此 $M_{z^{k}} A \neq A M_{z^{k}}$. 但是 $A$ 和 $M_{z^{k}}$ 都在 $\mathcal{A}^{\prime}\left(M_{f}\right)$ 中, 得出矛盾.

(iv) $\Rightarrow$ (v). 对 $z \in D_{1} \backslash \Gamma$, 因为

$$
\begin{gathered}
\alpha_{1}(z)+\alpha_{2}(z)+\cdots+\alpha_{n}(z)=h_{1}(z), \\
h_{k+1}=z^{k} h_{1}, \quad 1 \leqslant k \leqslant n-1,
\end{gathered}
$$

由 (iv) 可得

$$
\left\{\begin{array}{l}
\left(Z_{1}(z)-z\right) \alpha_{2}(z)+\left(Z_{2}(z)-z\right) \alpha_{3}(z)+\cdots+\left(Z_{n-1}(z)-z\right) \alpha_{n}(z)=0, \\
\left(Z_{1}^{2}(z)-z^{2}\right) \alpha_{2}(z)+\left(Z_{2}^{2}(z)-z^{2}\right) \alpha_{3}(z)+\cdots+\left(Z_{n-1}^{2}(z)-z^{2}\right) \alpha_{n}(z)=0, \\
\cdots \\
\left(Z_{1}^{n-1}(z)-z^{n-1}\right) \alpha_{2}(z)+\left(Z_{2}^{n-1}(z)-z^{n-1}\right) \alpha_{3}(z)+\cdots+\left(Z_{n-1}^{n-1}(z)-z^{n-1}\right) \alpha_{n}(z)=0,
\end{array}\right.
$$

经计算可知系数行列式仍然是一个 Vandermonde 行列式:

$$
V=\left|\begin{array}{cccc}
Z_{1}-z & Z_{2}-z & \cdots & Z_{n-1}-z \\
Z_{1}^{2}-z^{2} & Z_{2}^{2}-z^{2} & \cdots & Z_{n-1}^{2}-z^{2} \\
\vdots & \vdots & & \vdots \\
Z_{1}^{n-1}-z^{n-1} & Z_{2}^{n-1}-z^{n-1} & \cdots & Z_{n-1}^{n-1}-z^{n-1}
\end{array}\right|
$$

SCIENCE IN CHINA Ser. A Mathematics 


$$
=(-1)^{n-1}\left|\begin{array}{ccccc}
1 & 1 & \cdots & 1 & 1 \\
Z_{1} & Z_{2} & \cdots & Z_{n-1} & z \\
Z_{1}^{2} & Z_{2}^{2} & \cdots & Z_{n-1}^{2} & z^{2} \\
\vdots & \vdots & & \vdots & \vdots \\
Z_{1}^{n-1} & Z_{2}^{n-1} & \cdots & Z_{n-1}^{n-1} & z^{n-1}
\end{array}\right| \neq 0
$$

因此 $\alpha_{2}(z)=\alpha_{3}(z)=\cdots=\alpha_{n}(z)=0$ 且 $\mathcal{A}^{\prime}\left(M_{f}\right)=\left\{M_{g}: g \in R(D)\right\}$. 因为 $\mathcal{A}^{\prime}\left(M_{f}\right)$ 中不包含非平凡的幂等元, 所以 $M_{f}$ 是强不可约的.

(v) $\Rightarrow$ (i). 设 $M_{f}^{*}$ 的极小指标为 $n$ 且 $n \geqslant 2$, 则由定理 2.2, 对任意 $A \in \mathcal{A}^{\prime}\left(M_{f}\right)$, $A g=\alpha_{1}(z) g(z)+\cdots+\alpha_{n}(z) g\left(Z_{n-1}(z)\right)$. 因为 $M_{z}^{*} \in \mathcal{A}^{\prime}\left(M_{f}^{*}\right)$, 与 $M_{z}$ 对应的函 数 $\alpha_{1}(z)=z, \alpha_{2}(z)=\cdots=\alpha_{n}(z)=0$, 因此对任意 $u \in \Lambda, M_{z}^{*} k_{u}=\bar{u} k_{u}$. 又 $M_{z}^{*} k_{Z_{1}(u)}=\overline{Z_{1}(u)} k_{Z_{1}(u)}$, 根据引理 2.5, $M_{z}^{*}(f(u))=\left.M_{z}^{*}\right|_{\operatorname{ker} M_{f-f(u)}^{*}}$ 的谱是连通 的, 从而 $u=Z_{1}(u)$. 这与 $z \in \Lambda$ 时 $z \neq Z_{z}$ 矛盾, 因此 $n=1$ 且 $M_{f}^{*} \in B_{1}(\Omega)$.

例 2.7 设 $f(z)=z^{3}-2 z^{2}+\frac{4}{3} z$, 则 $M_{f}^{*} \in B_{1}\left(\Omega_{1}\right) \cap B_{2}\left(\Omega_{2}\right) \cap B_{3}\left(\Omega_{3}\right)$, 而 $\mathcal{A}^{\prime}\left(M_{f}\right)=\left\{M_{g}: g \in R(D)\right\}$, 其中

$$
\begin{aligned}
& D_{1}=f^{-1}\left(\Omega_{1}\right)=D \backslash\left(S_{1} \cup S_{2}\right), \\
& D_{2}=f^{-1}\left(\Omega_{2}\right)=\left[\left(S_{1} \cup S_{2}\right) \backslash\left(S_{1} \cap S_{2}\right)\right], \\
& D_{3}=f^{-1}\left(\Omega_{3}\right)=S_{1} \cap S_{2},
\end{aligned}
$$

而

$$
\begin{aligned}
& S_{1}=\left\{z \in D:\left|z-\left(1+\frac{\sqrt{3}}{3} \mathrm{i}\right)\right|<1\right\}, \\
& S_{2}=\left\{z \in D:\left|z-\left(1-\frac{\sqrt{3}}{3} \mathrm{i}\right)\right|<1\right\} .
\end{aligned}
$$

当 $z \in D_{2}$ 时,

当 $z \in D_{3}$ 时,

$$
Z_{1}(z)= \begin{cases}\left(-\frac{1}{2}+\frac{\sqrt{3}}{2} \mathrm{i}\right) z+1-\frac{\sqrt{3}}{3} \mathrm{i}, & z \in S_{1} \backslash S_{2} \\ \left(-\frac{1}{2}-\frac{\sqrt{3}}{2} \mathrm{i}\right) z+1+\frac{\sqrt{3}}{3} \mathrm{i}, & z \in S_{2} \backslash S_{1}\end{cases}
$$

$$
\begin{aligned}
& Z_{1}(z)=\left(-\frac{1}{2}+\frac{\sqrt{3}}{2} \mathrm{i}\right) z+1-\frac{\sqrt{3}}{3} \mathrm{i} \\
& Z_{2}(z)=\left(-\frac{1}{2}-\frac{\sqrt{3}}{2} \mathrm{i}\right) z+1+\frac{\sqrt{3}}{3} \mathrm{i} .
\end{aligned}
$$

在本例中, $M_{f}^{*} \in B_{1}\left(\Omega_{1}\right) \cap B_{2}\left(\Omega_{2}\right) \cap B_{3}\left(\Omega_{3}\right)$, 因此它的极小指标为 1 .

对 $n \geqslant 2$, 记 $\omega$ 为 1 的 $n$ 次方根, 即 $\omega \in \mathbb{C}$ 满足 $\omega^{n}=1$. 记 $\Delta_{n}$ 为 $n$ 阶 
Vandermonde 行列式:

$$
\Delta_{n}=\left|\begin{array}{ccccc}
1 & 1 & 1 & \cdots & 1 \\
1 & \omega & \omega^{2} & \cdots & \omega^{n-1} \\
\vdots & \vdots & \vdots & & \vdots \\
1 & \omega^{n-1} & \omega^{2(n-1)} & \cdots & \omega^{(n-1)^{2}}
\end{array}\right|
$$

对 $1 \leqslant i, j \leqslant n$, 记 $\Delta_{i j}$ 为 $\Delta_{n}$ 的 $(i, j)$ 元素的代数余子式.

命题 2.8 算子 $A \in \mathcal{A}^{\prime}\left(M_{z^{n}}\right)$ 的充分必要条件是对任意 $g \in R(D)$, 当 $z \neq 0$ 时,

$$
(A g)(z)=\sum_{i=1}^{n} \alpha_{i}(z) g\left(\omega^{i-1} z\right)
$$

其中 $\alpha_{i}(z)=\left(\sum_{j=1}^{n} \Delta_{i j} \frac{h_{j}}{z^{j-1}}\right) / \Delta_{n},\left\{h_{j}\right\}_{j=1}^{n}$ 是 $R(D)$ 中的 $n$ 个函数.

证 由定理 2.2, 当 $z, z_{0} \in D \backslash\{0\}$ 时,

$$
z^{n}-z_{0}^{n}=\left(z-z_{0}\right)\left(z-\omega z_{0}\right) \cdots\left(z-\omega^{n-1} z_{0}\right), \quad Z_{i}\left(z_{0}\right)=\omega^{i-1} z_{0} .
$$

设 $A \in \mathcal{A}^{\prime}\left(M_{z^{n}}\right)$, 记 $h_{k}=A z^{k-1}$, 利用定理 2.2, 经计算可知

$$
\alpha_{i}(z)=\frac{1}{\Delta_{n} z^{\frac{n(n+1)}{2}}}\left[\sum_{j=1}^{n} \Delta_{i j} z^{\frac{n(n+1)}{2}-j+1} h_{j}(z)\right]=\frac{1}{\Delta_{n}}\left[\sum_{j=1}^{n} \Delta_{i j} \frac{h_{j}(z)}{z^{j-1}}\right] .
$$

另一方面, 对任意 $h_{1}, h_{2}, \cdots, h_{n} \in R(D)$, 令

$$
\alpha_{i}(z)=\frac{1}{\Delta_{n}} \sum_{j=1}^{n} \Delta_{i j} \frac{h_{j}(z)}{z^{j-1}}, \quad z \neq 0, \quad 1 \leqslant i \leqslant n .
$$

对 $g \in R(D)$, 形式上令

$$
(A g)(z)=\sum_{i=1}^{n} \alpha_{i}(z) g\left(\omega^{i-1} z\right) \quad(z \neq 0) .
$$

下面证 $A$ 是有界的.

对自然数 $m$, 记 $\bar{m} \equiv m(\bmod n)$ 且 $0 \leqslant \bar{m} \leqslant n-1$.

设 $g(z)=\sum_{m=0}^{\infty} g_{m} z^{m}$, 则

$$
\begin{aligned}
(A g)(z) & =\sum_{i=1}^{n} \alpha_{i}(z) g\left(\omega^{i-1} z\right) \\
& =\frac{1}{\Delta_{n}} \sum_{i=1}^{n}\left[\left(\sum_{j=1}^{n} \Delta_{i j} \frac{h_{j}(z)}{z^{j-1}}\right)\left(\sum_{m=0}^{\infty} g_{m} \omega^{m(i-1)} z^{m}\right)\right] \\
& =\frac{1}{\Delta_{n}} \sum_{i=1}^{n}\left[\sum_{m=0}^{\infty} g_{n}\left(\sum_{j=1}^{n} \Delta_{i j} \omega^{\bar{m}(i-1)} h_{j} z^{m-j+1}\right)\right] \\
& =\frac{1}{\Delta_{n}} \sum_{m=0}^{\infty} g_{m}\left[\sum_{i=1}^{n}\left(\sum_{j=1}^{n} \Delta_{i j} \omega^{\bar{m}(i-1)} h_{j} z^{m-j+1}\right)\right]
\end{aligned}
$$

SCIENCE IN CHINA Ser. A Mathematics 


$$
=\frac{1}{\Delta_{n}} \sum_{m=0}^{\infty} g_{m}\left[\sum_{j=1}^{n}\left(\sum_{i=1}^{n} \Delta_{i j} \omega^{\bar{m}(i-1)} h_{j} z^{m-j+1}\right)\right] .
$$

由行列式的性质, 经计算得

$$
\sum_{i=1}^{n} \Delta_{i j} \omega^{\bar{m}(i-1)}= \begin{cases}\Delta_{n}, & \bar{m}=j-1 \\ 0, & \bar{m} \neq j-1\end{cases}
$$

因此

$$
\begin{aligned}
(A g)(z) & =\sum_{k=0}^{\infty}\left(g_{k n} z^{k n} h_{1}(z)+g_{k n+1} z^{k n} h_{2}(z)+\cdots+g_{k n+n-1} z^{k n} h_{n}\right) \\
& =\sum_{i=0}^{n}\left(\sum_{k=0}^{\infty} g_{k n+i-1} z^{k n} h_{i}\right) .
\end{aligned}
$$

对任意 $i(1 \leqslant i \leqslant n)$,

$$
\begin{aligned}
\left\|\sum_{k=0}^{\infty} g_{k n+i-1} z^{k n} h_{i}\right\|^{2} & \leqslant M\left\|h_{i}\right\|^{2}\left\|\sum_{k=0}^{\infty} g_{k n+i-1} z^{k n}\right\|^{2} \\
& =M\left\|h_{i}\right\|^{2} \sum_{k=0}^{\infty}\left|\frac{g_{k n+i-1}}{\beta_{k n+i-1}} \frac{\beta_{k n+i-1}}{\beta_{k n}} e_{k n}\right|^{2} \\
& =M\left\|h_{i}\right\|^{2} \sum_{k=0}^{\infty}\left|\frac{g_{k n+i-1}}{\beta_{k n+i-1}}\right|^{2}\left|\frac{\beta_{k n+i-1}}{\beta_{k n}}\right|^{2} \\
& \leqslant N\|g\|^{2},
\end{aligned}
$$

所以 $\|A g\|^{2} \leqslant n N\|g\|^{2}$, 即 $A$ 是有界线性算子. 通过计算可以验证 $A \in \mathcal{A}^{\prime}\left(M_{f}\right)$.

对任意算子 $T, \mathcal{A}^{\prime}(T)$ 中的幂等元 $P$ 都对应了空间 $H$ 关于 $T$ 的一个 Banach 约化分解: $H=H_{1} \dot{+} H_{2}$, 其中 $H_{1}=\operatorname{ran} P, H_{2}=\operatorname{ran}(1-P)$.

下面的命题刻画了 $M_{z^{2}}$ 的所有的 Banach 约化分解:

命题 2.9 设 $P \in \mathcal{A}^{\prime}\left(M_{z^{2}}\right)$, 则下列叙述等价:

(i) $P^{2}=P$.

(ii) 对任意 $g \in R(D), P g=\alpha_{1}(z) g(z)+\alpha_{2}(z) g(-z)(z \neq 0)$, 且下列两者之 一成立:

(a) $a_{2}(z) \equiv 0$ 且 $P=I$ 或 0 ;

(b) $\alpha_{1}(z)=\frac{a_{-1}}{z}+\frac{1}{2}+\sum_{k=0}^{\infty} a_{2 k+1} z^{2 k+1}$ 且 $\alpha_{1}(z) \alpha_{1}(-z)=\alpha_{2}(z) \alpha_{2}(-z)$.

(iii) 若 $h_{1}, h_{2} \in R(D), h_{1}(z)=\sum_{n=0}^{\infty} b_{n} z^{n}, h_{2}(z)=\sum_{n=0}^{\infty} c_{n} z^{n}$, 则下列两者 之一成立:

(a) $z h_{1}(z)=h_{2}(z)$ 且 $P=I$ 或 0 ;

(b) $b_{0}+c_{1}=1 / 2, b_{2 k}=-c_{2 k+1}(k \geqslant 1)$ 且 $h_{1}(z) h_{2}(-z)=h_{1}(-z) h_{2}(z)$.

证 (i) $\Rightarrow$ (ii). 设 $P \in \mathcal{A}^{\prime}\left(M_{z^{2}}\right)$, 则由命题 2.8, 对任意 $g \in R(D)$, 有

$$
P g=\alpha_{1}(z) g(z)+\alpha_{2}(z) g(-z) \quad(z \neq 0) .
$$


由于 $P^{2}=P$, 故

$$
\begin{aligned}
& \alpha_{1}^{2}(z) g(z)+\alpha_{1}(z) \alpha_{2}(z) g(-z)+\alpha_{2}(z) \alpha_{1}(-z) g(-z)+\alpha_{2}(z) \alpha_{2}(-z) g(z) \\
= & \alpha_{1}(z) g(z)+\alpha_{2}(z) g(-z) \quad(z \neq 0) .
\end{aligned}
$$

当 $g=e$ 时,

$$
\alpha_{1}^{2}(z)+\alpha_{1}(z) \alpha_{2}(z)+\alpha_{2}(z) \alpha_{1}(-z)+\alpha_{2}(z) \alpha_{2}(-z)=\alpha_{1}(z)+\alpha_{2}(z) ;
$$

当 $g(z)=z$ 时,

$$
\alpha_{1}^{2}(z)-\alpha_{1}(z) \alpha_{2}(z)-\alpha_{2}(z) \alpha_{1}(-z)+\alpha_{2}(z) \alpha_{2}(-z)=\alpha_{1}(z)-\alpha_{2}(z) .
$$

计算可得

$$
\begin{gathered}
\alpha_{1}^{2}(z)+\alpha_{2}(z) \alpha_{2}(-z)=\alpha_{1}(z), \\
{\left[\alpha_{1}(z)+\alpha_{1}(-z)\right] \alpha_{2}(z)=\alpha_{2}(z) .}
\end{gathered}
$$

由于 $\alpha_{1}$ 和 $\alpha_{2}$ 解析, $\alpha_{2}(z) \equiv 0$ 或 $\alpha_{1}(z)+\alpha_{1}(-z) \equiv 1$.

当 $\alpha_{2}(z) \equiv 0$ 时, $\alpha_{1}(z) \equiv 1$ 或 0 , 此时 $P=I$ 或 $P=0$;

当 $\alpha_{1}(z)+\alpha_{1}(-z) \equiv 1$ 时, 由命题 $2.8, \alpha_{1}(z)$ 在 $z=0$ 处有一级极点, 在 $D \backslash\{0\}$ 内解析, 因此, $\alpha_{1}(z)$ 可以表示为

$$
\alpha_{1}(z)=\frac{a_{-1}}{z}+\frac{1}{2}+\sum_{k=0}^{\infty} a_{2 k+1} z^{2 k+1} .
$$

由 (5) 式,

$$
\begin{aligned}
\alpha_{2}(z) \alpha_{2}(-z) & =\alpha_{1}(z)\left[1-\alpha_{1}(z)\right] \\
& =\alpha_{1}(z)\left[-\frac{a_{-1}}{z}+\frac{1}{2}-\sum_{k=0}^{\infty} a_{2 k+1} z^{2 k+1}\right] \\
& =\alpha_{1}(z) \alpha_{1}(-z) .
\end{aligned}
$$

(ii) $\Rightarrow$ (i).

$$
\begin{aligned}
\left(P^{2} g\right)(z) & =\alpha_{1}^{2}(z) g(z)+\alpha_{2}(z) \alpha_{2}(-z) g(z)+\alpha_{2}(z) g(-z)\left[\alpha_{1}(z)+\alpha_{1}(-z)\right] \\
& =\alpha_{1}^{2}(z) g(z)+\alpha_{1}(z) \alpha_{1}(-z) g(z)+\alpha_{2}(z) g(-z) \\
& =\alpha_{1}(z) g(z)\left[\alpha_{1}(z)+\alpha_{1}(-z)\right]+\alpha_{2}(z) g(-z) \\
& =\alpha_{1}(z) g(z)+\alpha_{2}(z) g(-z)=(P g)(z) .
\end{aligned}
$$

经计算可证明 (ii) $\Leftrightarrow$ (iii).

\section{参考文献}

1 Dixmier J, Foiaç C. Surle Spectre Ponctuel d'un Opératear. Colloq Math János Bolyai. 5. Hilbert Space Operators and Operator Algebras. Amsterdam-London: North Holland Publ Co, 1972. 127 133

2 Herrero D A, Taylor T J, Wang Z Y. Variation of the point spectrum under compact perturbations. Operator Theory: Advances and Applications, 1988, 32: 113 158 
3 Wang Zongyao. Multiplication operator on Sobolev space. 见: 中国博士后首届学术大会论文集. 北 京: 国防工业出版社, 1992. 1167 1170

4 Jiang C L, Wang Z Y. A class of strongly irreducible operators with nice property. J Operator Theory, 1996, 36: 3 19

5 Jiang C L, Wang Z Y. Strongly Irreducible Operators on Hilbert Space. Pitman Research Notes in Mathematics Series 389. Essex: Longman, 1998

6 Adams R A. Sobolev Spaces. New York-San Francisco-London: Academic Press, 1975

7 Lambert A. Strictly cyclic operator algebra. Pacific J Math, 1971, 38: 717 766

8 Rudin W. Real and Complex Analysis. New York: McGraw-Hill Book Company, 1974

9 Conway J B, Herrero D A, Morrel B B. Completing the Riesz-Dunford Functional Calculus. Memoirs of AMS, Vol 82, No 417. Providence: Amer Math Soc, 1989

10 Cowen M J, Douglas R G. Complex geometry and operator theory. Bull Amer Math Soc, 1977, 83: $131 \sim 133$

11 Griffiths P. 代数曲线. 北京: 北京大学出版社, 1985

12 Jiang C L. Similarity classification of Cowen-Douglas operators. Canad J Math, 2004, 56: 742 775 